\title{
Institutionelle Bedingungen kollektiver Handlungsfähigkeit im urbanen Raum: Eine QCA von siebzehn europäischen Entscheidungsfällen
}

\author{
Fritz Sager
}

\begin{abstract}
Zusammenfassung: Der vorliegende Aufsatz fragt nach den institutionellen Bedingungen für die Bewältigung von Interdependenzproblemen im urbanen Raum. Als Messgrösse für kollektive Handlungsfähigkeit wird die Qualität von Politikkoordination im Prozess und im Entscheidungsergebnis gewählt. Für die empirische Untersuchung werden zwei einander entgegen gesetzte verwaltungswissenschaftliche Modelle metropolitaner Institutionen hergeleitet und Hypothesen formuliert: Einerseits steht das neoprogressive Modell für Zentralisierung, konsolidierte Raumstrukturen sowie eine professionelle und politisch unabhängige Verwaltung. Andererseits steht das Public Choice-Modell für Dezentralisierung, fragmentierte Raumstrukturen sowie eine unprofessionelle und politisch abhängige Verwaltung. Die Resultate des Vergleichs von siebzehn Fallstudien zu raumwirksamen Entscheidungsprozessen in westeuropäischen Stadträumen mithilfe Qualitative Comparative Analysis (QCA) weisen die neoprogressiven Postulate als leistungsfähiger für Politikkoordination aus als das Public Choice-Modell.
\end{abstract}

Stichworte: Qualitative Comparative Analysis (QCA) · urbane Räume · Politikkoordination · Neo-Institutionalismus · kollektive Handlungsfähigkeit

\begin{abstract}
Institutional Conditions for Collective Capacity to Act in the Urban Space: A QCA of Seventeen European Decision Cases. The present article questions the institutional preconditions for solving problems of interdependency in urban areas. Policy coordination in terms of processes as well as outcomes serves as an indicator for the collective capacity to act. Hypotheses are derived from two institutionalist schools: first, from the neoprogressive model that stands for direct public service provision by centralized and professionalised bureaucracies within consolidated municipalities, and second, from the public choice model that represents a decentralized, non-professional,
\end{abstract}

Prof. Dr. Fritz Sager $(\bowtie)$

Assistant Professor for Policy Analysis and Evaluation, Institute of Political Science, University of Bern

Unitobler - Lerchenweg 36, CH-3000 Bern 9, Switzerland

Tel:. ++41 31 631-3285, Fax: ++41 31 631-8590

E-Mail: sager@ipw.unibe.ch 
and politically dependent administration in fragmented urban areas. The results of the comparison of seventeen case studies regarding the integration of urban transport and land use policies in Western European urban areas employing Qualitative Comparative Analysis (QCA) show that well co-ordinated policy decisions are only implemented in institutional settings that largely correspond to the neoprogressive model.

Keywords: Qualitative Comparative Analysis (QCA) · urban areas · policy coordination $\cdot$ neo-institutionalism $\cdot$ collective capacity to act

\section{Einleitung}

Urbane Räume in Westeuropa sind zunehmend einem doppelten politischen Problemdruck ausgesetzt. Einerseits besteht ein Trend zur großräumigen ökonomischen und demografischen Konzentration in so genannten Metropolitanräumen. Diese Metropolitanräume gewinnen aufgrund des internationalen Wettbewerbs an wirtschaftlicher, sozialer und kultureller Bedeutung. Die formal-politischen Problemlösungskompetenzen dieser urbanen Großräume hinsichtlich Politikformulierung und -umsetzung entsprechen aber kaum ihrem Problemdruck. Eine Lösung kann in einem geeinten Auftreten der Agglomerationsräume gegenüber den übergeordneten staatlichen Ebenen gesehen werden. Andererseits verstärkt sich aber innerhalb der urbanen Großräume die Fragmentierungstendenz, die eine notwendige und auch geforderte innere Einheit verhindern kann. Die Abwanderung aus den Kernstädten zeichnet sich vielmehr durch gerade gegenläufige, sich selbst verstärkende Mechanismen aus, die einen Kreislauf der Zersiedelung etabliert haben: Je mehr Menschen in die Vorortsgemeinden abwandern, desto unattraktiver werden die Kerngemeinden, desto mehr Menschen wandern ab. Die urbanen Räume sind aufgrund dieser partikulären Stellung vor einen besonderen Handlungsbedarf gestellt. Die Kumulation der Probleme innerhalb der Metropolitanräume droht gekoppelt mit der Entwicklung großräumiger Machtzentren und einer wachsenden internationalen Städtekonkurrenz zu einer Pattsituation zu führen, die den urbanen Handlungsspielraum von innen ebenso wie von außen blockiert. Als Ausweg wird generell die querschnittsorientierte Zusammenarbeit von Stadt und Region, die auf die Koordination zwischen politischen Programmen aus verschiedenen Politikbereichen abzielt, gesehen (Benz 1994; Blatter 2005; Heinz 2000; Le Galès 1998; Sager 2002).

Kernstadt und Umlandgemeinden sehen sich damit in großem Maße dem Problem kollektiver Handlungsfähigkeit gegenüber gestellt. Dabei bedarf es insbesondere „solche(r) Modi der Interdependenzbewältigung, die auf die Herstellung und Stabilisierung kollektiver Handlungsfähigkeit einer Mehrzahl individueller Akteure hinauslaufen" (Schimank 2002: 31). Im Folgenden wird argumentiert, dass Politikkoordination ein geeignetes Maß kollektiver Handlungsfähigkeit darstellt. Zur Erklärung von Politikkoordination werden aus in zwei unterschiedlichen intellektuellen Traditionen wurzelnden, verwaltungswissenschaftlichen Schulen metropolitaner Institutionen Hypothesen abgeleitetet, die in Konkurrenz zueinander stehen. Die beiden Schulen sind das der Bürokratieanalyse von Weber (1972) verwandte und dem soziologischen , bounded rationality'-Institutionalismus (Jones 2003) zuzurechnende neoprogressive Mo- 
dell und das dem ökonomischen Institutionalismus zugehörige Public Choice-Modell (Ostrom/Ostrom 1971). Der Fokus der Analyse liegt bei den Strukturen der öffentlichen Verwaltung als vordringlicher Form sozialer Institutionen der modernen Gesellschaft (Zucker 1983: 1).

Der Aufsatz ist wie folgt aufgebaut: Im nächsten Abschnitt werden die beiden Ansätze der neoprogressiven und der Public Choice-Schule ideentheoretisch verortet und diskutiert. Im nachfolgenden Abschnitt wird das Problem der Politikkoordination als Frage kollektiver Handlungsfähigkeit und als abhängige Größe für die empirische Analyse eingeführt und anschließend werden die Hypothesen präsentiert. Der nächste Abschnitt stellt die Forschungsstrategie, die Wahl der Fälle, die Methode für die empirische Untersuchung sowie die Operationalisierung der Variablen dar. Die anschließende empirische Analyse besteht aus dem systematischen Vergleich von siebzehn Fallstudien zur Politikkoordination in urbanen Räumen Westeuropas. Hierzu wird auf die makro-qualitative Methode der Qualitative Comparative Analysis (QCA) zurückgegriffen. ${ }^{1}$ Diese Methode bietet sich für die vorliegende Untersuchung an, da sie einerseits für mittlere Fallzahlen besonders geeignet ist und andererseits das Augenmerk nicht auf isolierte Wirkungsweisen einzelner Variablen bzw. - in der QCA-Terminologie - Bedingungen, sondern auf die kombinierten Effekte unterschiedlicher Ausprägungen von Bedingungen legt. Die Schlussfolgerungen bestehen aus einer zusammenfassenden Würdigung der Erkenntnisse.

\section{Zwei Modelle metropolitaner Institutionen}

Wie Krücken (2002: 227) feststellt, lässt sich seit den 1980er Jahren „,eine Wiederentdeckung institutionalistischen Gedankenguts innerhalb des disziplinären Dreiecks von Soziologie, Politik- und Wirtschaftswissenschaften beobachten." Dieser Neo-Institutionalismus ist an der Schnittstelle von Organisations-, Wirtschafts- und Netzwerkforschung angesiedelt (Powell/DiMaggio 1991). Die vorliegende Studie bedient sich dieser Strömung, die ,in der aktuellen Forschung in erster Linie als Generator von Theorien mittlerer Reichweite dient" (Krücken 2002), indem sie nach den institutionellen Bedingungen für erfolgreiche Politikkoordination fragt. Basierend auf den Arbeiten von Scott und Meyer (1991) können vier zentrale Organisationsdimensionen identifiziert werden, die relevant sind für das soziale und politische Verhalten sowie die politische Entscheidungsfindung innerhalb von Organisationen: (1) Zentralisierung vs. Dezentralisierung, (2) räumliche Konsolidierung vs. räumliche Fragmentierung, (3) Professionalisierung vs. nicht professionelle Verwaltung und (4) autonome vs. politisch abhängige Verwaltung.

1 Obwohl die QCA-Methode in der internationalen Forschung einen wachsenden Anwenderkreis findet (vgl. die Internetplattform auf http://compasss.org/), gibt es im deutschen Sprachraum bislang nur wenige Untersuchungen, die sich der Vorzüge dieses Ansatzes bedienen (Berg-Schlosser und Quenter 1996, Obinger 1998, Sager 2002). Die Diskussion beschränkt sich daneben auf Abhandlungen in methodischen Lehrbüchern (Berg-Schlosser 1997, Wagschal 1999, Schneider und Wagemann 2007). 
Entlang dieser vier Analysedimensionen werden im Folgenden zwei institutionelle Designs aus der Literatur heraus erarbeitet, die auf Basis der beiden Schulen der Progressive Reformers und des Public Choice postuliert werden können.

\section{Neoprogressives Modell: Zentralisierung, Konsolidierung, professionelle und politisch unabhängige Verwaltung}

Die u.s.-amerikanische Schule der Progressive Reformers entstand aus dem Unmut über die korrupten Regime in den amerikanischen Städten des ausgehenden 19. Jahrhunderts heraus. Das Wiederaufkommen von Postulaten des Progressive Movement in der verwaltungswissenschaftlichen Diskussion in den 1990er Jahren (Hill 1991; Goodsell 1994) war eine Reaktion auf den Siegeszug des Public Choice-Ansatzes. Die Vertreter der von Lowery (1999) neu mit dem Label ,neoprogressiv“ versehenen Schule begegnen vor allem dem grundsätzlichen Public Choice-Argument des Staatsversagens mit der Erkenntnis, dass bei verschiedenen öffentlichen Aufgaben die Marktmechanismen versagen. Neuere neoprogressive Arbeiten anerkennen sowohl die theoretischen Argumente wie auch die empirischen Leistungsausweise des Public Choice-Modells und stehen für eine differenzierte Auseinandersetzung ein, die auf eine eigentliche Neukonzipierung des Verwaltungsmodells des Progressive Movement hinausläuft (Lowery 1999: 47, Sager 2005).

Das institutionelle Modell, das die neoprogressive Schule vertritt, kann entlang der Analysedimensionen von Scott und Meyer (1991) mit den folgenden vier Postulaten beschrieben werden: Zentralisierung, Konsolidierung, Professionalisierung und Autonomie.

- Das Postulat der Zentralisierung entspricht in seinen Grundzügen dem Weberschen Bürokratiemodell mit dem Element des hierarchischen Aufbaus (Weber 1972). Indem die übergeordneten Stellen die nachgeordneten kontrollieren und Leitungsorgane monokratisch und nicht kollegial aufgebaut sind, besteht eine Tendenz zur Zentralisierung. Durch diese Intensivierung der organisatorischen Kontrollen werden einheitlichere und berechenbarere Leistungen erzeugt und die arbeitsteilige Differenzierung gefördert. Dieses System verbessert die synoptische Kapazität der Verwaltung, verhindert Beamtenwillkür auf den verschiedenen Stufen und steigert so durch Skaleneffekte und wegfallende Koordinationskosten die Effizienz öffentlichen Handelns (Nigro/Nigro 1989: 110).

- Das Argument der räumlichen Konsolidierung ist zentral in der neoprogressiven Argumentation. Keating (1995: 118-123) nennt vier Argumentationslinien: (1) Effizienz, (2) Demokratie, (3) Verteilungsequität und (4) urbane Entwicklung. Das Argument der Effizienz basiert vor allem auf einer ökonomischen Sichtweise. Für Konsolidierung sprechen dabei Argumente der minimalen Projektgröße, anfallende Koordinationskosten bei fragmentierten Gebieten und sinkende Durchschnittskosten (zur Transaktionskostentheorie vgl. Wittek/Flache 2002: 62). Das Demokratieargument besagt einerseits, dass mit großen Gebietseinheiten ein stärkeres Auftreten gegenüber übergeordneten staatlichen Ebenen möglich wird. Andererseits kommen Lyons et al. (1992) in einer vergleichenden Analyse zum Schluss, dass Minderheiten 
in konsolidierten Gebieten stärker partizipieren und besser informiert sind über die politischen Strukturen ihrer Gemeinde als Bewohner und Bewohnerinnen fragmentierter Systeme. Das Distributionsargument postuliert, dass konsolidierte Gebietseinheiten zu einer besseren Verteilungsgerechtigkeit und somit auch zu einer grösseren Zufriedenheit bei ihrer Bevölkerung führen, als das in fragmentierten Agglomerationen möglich ist (Lyons et al. 1992). Das Entwicklungsargument schliesslich besagt, dass konsolidierte Gebietseinheiten die Konkurrenzfähigkeit des urbanen Gesamtgebiets erhöhen und so die wirtschaftliche Entwicklung gewährleisten.

- Das Postulat der Professionalisierung gehört zum Grundstock des Technik- und Modernisierungsglaubens der Progressive Reformers (West 1995: 5). Er manifestierte sich von Beginn weg in der Forderung, gut ausgebildetes und spezialisiertes Fachwissen in den Dienst der Gesellschaft zu stellen bzw. in die Verwaltung einzubinden. Der funktionalen Ausdifferenzierung nach den Kriterien der professionellen Spezialisierung und Ausdifferenzierung werden in der Planungsliteratur der 1960er Jahre weitere Vorteile hinsichtlich der kognitiven Voraussetzungen für kooperatives Handeln zugesprochen (Nigro/Nigro 1989: 110; Simon 1962). Dabei kann auf das - ursprünglich für die Analyse internationaler Verhandlungen entwickelte - Konzept der „epistemic communities“ (Haas 1964) zurückgegriffen werden. Als „epistemic community“ werden Netzwerke von professionellen Experten in bestimmten Politikfeldern bezeichnet, deren Wissen für bestimmte Politikinhalte von zentraler Relevanz ist (Haas 1992: 3). Das durch gemeinsame Ausbildung und professionelle Werthaltungen generierte und reproduzierte Wissen führt zu einem grenzüberschreitenden, sektoralen Konsens dieser Akteure. Da das verfolgte Ziel ein rationales Politikergebnis und nicht die Befriedigung von Partikularinteressen ist, führen solche professionellen Werthaltungen zu einer konsensuellen grenzüberschreitenden Politikkoordination.

- Das Postulat der politischen Unabhängigkeit wurzelt in einer der Gründerschriften des Progressive Movements, in welcher Wilson (1887) als Wurzel von Korruption und Ungerechtigkeit die fehlende Trennung von politischer und administrativer Sphäre ausmachte. Der Begriff der Autonomie wird hier auf die politische Verwaltung als eigenständigen Verhandlungspartner im politischen Prozess bezogen. Autonomie meint dabei entsprechend die der politischen Verwaltung eigenen politischen Handlungsspielräume, die durch eine klare Kompetenzabgrenzung von der Legislative definiert sind. Es handelt sich also um eine begrenzte politische Aktionsfähigkeit, die vor Direkteinwirkungen von außen geschützt ist. Solche Interventionen können nicht allein durch Einzeleingriffe der Legislative erfolgen, sondern auch durch den Einfluss ökonomischer und sozialer Einzelinteressen oder durch die Partizipation von Politikbetroffenen. Eine hohe Autonomie der Verwaltung wirkt der Neutralisierung des Expertenwissens durch politische Einflussnahme entgegen und ermöglicht die problembezogene Abstimmung öffentlichen Handelns (Wolf 1993).

In umfassendem Gegensatz zu diesen Postulaten steht das Modell der Public ChoiceSchule, das in den folgenden Abschnitten präsentiert wird. 
Public Choice: Dezentralisierung, Fragmentierung, nicht professionelle und politisch abhängige Verwaltung

1971 veröffentlichten Elinor und Vincent Ostrom ihren Aufsatz „Public Choice: A Different Approach to Public Administration" (Ostrom/Ostrom 1971) und brachen damit mit der fast hundert Jahre alten progressiven Dominanz in der amerikanischen Verwaltungslehre. Das neu postulierte, polit-ökonomische Verständnis von Verwaltung war von neuen Paradigmen getragen, die Teil des umfassenderen sozialwissenschaftlichen Konzepts der Rational Choice-Theorie waren, die ,in ihrer orthodoxen Form für die Organisationsforschung ein radikales und provozierendes Programm" (Wittek/Flache 2002: 55, auch Hay 2004) darstellte.

Die grundsätzliche Argumentationslinie des Public Choice-Modells der Verwaltung lässt sich entlang der Prämissen der ökonomischen Theorie der Politik (Downs 1957) wie folgt zusammenfassen: Jede Form staatlicher Intervention in die Gesellschaft ist eine Behinderung der Entfaltung der freien Marktwirtschaft und steht so dem grundlegenden Recht des Individuums auf Nutzenmaximierung entgegen. Da dem Individualitätsprinzip entsprechend die Wohlfahrt der Gesamtgesellschaft der Summe allen individuellen Wohlergehens durch rationale Nutzenmaximierung entspricht, wird diese Einschränkung negativ gewertet. Die Verwaltung ist die zentrale Trägerin staatlicher Interventionen und widerspricht deshalb schon von ihrer Grundidee her dem Prinzip des freien Marktes. Vielmehr verstärkt sie staatliche Eingriffe noch aufgrund eines systemimmanenten Teufelskreises, wollen doch die einzelnen Beamtinnen und Beamten dem Eigennutzaxiom entsprechend primär ihre Budgets maximieren und sorgen damit für ein stetiges Anwachsen der Staatsquote und somit auch des Interventionismus beziehungsweise der Rückbindung der individuellen Handlungsfreiheit (Niskanen 1971). Die öffentliche Verwaltung versagt so als System nicht nur in ihrer Aufgabe, die Bedürfnisse der Bürgerinnen und Bürger zu befriedigen (Staatsversagen), sondern ist zugleich auch undemokratisch. Generell steht der Public Choice-Ansatz also für eine möglichst klein gehaltene Verwaltung ein, um so die Behinderung des Marktes zu vereiteln. Das Postulat, dass entsprechende Organisationsstrukturen zu Koordination führen, entspringt dem Argument, dass das individuelle Streben nach der bestmöglichen Erreichung der persönlichen Ziele ,dann zu Kooperation führen (kann), wenn individuelle soziale Akteure voneinander funktional abhängig sind, sie also einander brauchen, um ihre persönlichen Ziele zu erreichen. (...) rational handelnde Individuen kooperieren, weil sie damit Verpflichtungen erzeugen, die sie später gemäß des Reziprozitätsprinzips wieder einfordern können“ (Wittek/Flache 2002: 75).

Das institutionelle Modell, das die Public Choice-Schule innerhalb dieser Vorgabe vertritt, kann entlang der gewählten Analysedimensionen mit den folgenden zentralen Postulaten charakterisiert werden: Dezentralisierung, Fragmentierung, Generalistenverwaltung und direkte politische Abhängigkeit.

- Mit der Dezentralisierung von Organisationsstrukturen, die verbunden ist mit einer Erhöhung der Autonomie der so geschaffenen Einheiten, wird mehr Eigenständigkeit und Verantwortlichkeit an die ,Front' delegiert, wo die Orientierung am Bürger beziehungsweise Kunden ungleich größer ist als bei der Zentralverwaltung. Der Ver- 
lust an operativen Steuerungs- und Kontrollmöglichkeiten wird kompensiert durch eine Verstärkung des strategischen Managements und entsprechende Qualitätsvorgaben durch die politisch verantwortlichen Stellen. Die Dezentralisation hat dabei den Vorteil, dass sie zu einer Vielzahl von Vergleichsgrößen führt, an denen die Leistungen der einzelnen Verwaltungseinheiten gemessen werden können (Schedler 1995: 95). Während die Vorteile für die Bürgerinnen und Bürger in der Verbesserung der individuellen Bedürfnisbefriedigung liegen, die nach ökonomischer Logik zur Steigerung des Gemeinwohls führt, fasst Budäus (1994: 56) die positiven Effekte, die sich für die öffentliche Verwaltung selber aus dezentralen Strukturen ergeben, wie folgt zusammen: Die Komplexität wird abgebaut und das Verwaltungshandeln wird transparenter, indem Leistungen und Kosten klar zugeordnet werden können. Die Zusammenfassung von Fach- und Ressourcenverantwortung führt zur Kongruenz von Entscheidung und Verantwortung für deren Folgewirkungen, wodurch innerhalb der Verwaltung wettbewerbsähnliche Koordinationsmechanismen institutionalisiert werden können.

- Eng mit dem Postulat der Dezentralisierung ist jenes der räumlichen Fragmentierung verbunden. Im Vordergrund steht das Effizienzargument. Tiebout (1956) versteht Lokalregierungen als Unternehmen und Bürger als Konsumenten. Entsprechend versuchen Lokalregierungen die Dienstleistungen, die von ihren Bürgern gewünscht werden, zu einem möglichst tiefen Preis anzubieten, während Bürger die politische Gebietseinheit mit der Mischung von Dienstleistungen und Steuerbelastung zu ihrer Wohngemeinde wählen, die am nächsten bei ihren Präferenzen liegt (Tiebout 1956: 420). Je grösser die Zahl der Anbieter von öffentlichen Gütern ist, desto eher entstehen Marktverhältnisse, die zu einer Befriedigung der individuellen Bedürfnisse und zu einer Effizienzsteigerung des Angebots durch Konkurrenz führen. Eine Schwäche des Modells von Tiebout (1956) liegt in seinen Grundannahmen, dass erstens Mobilität kostenlos ist und zweitens Arbeitsmärkte keine Rolle spielen. Weiterentwicklungen des Tiebout-Modells fragen entsprechend nach den Bedingungen, unter denen Individuen „can shop around for services“ (Tiebout 1956), ohne wegziehen zu müssen, und wie diese Bedingungen durch interkommunale Konkurrenz hergestellt werden können. Ostrom et al. (1988) argumentieren, dass gebietliche Fragmentierung und Wettbewerb zwischen Lokalregierungen die allokative Effizienz steigern, indem Kapital an jene Orte verschoben wird, an denen es am produktivsten eingesetzt werden kann. Ein zweites Argument des Public Choice-Modells ist die Stärkung des Demokratieprinzips im Sinne der unmittelbaren Befriedigung individueller politischer Präferenzen. Demokratie, so wird argumentiert, ist umso stärker, je näher die Politik bei der Bürgerin und beim Bürger ist.

- Das Postulat einer nicht professionellen Verwaltung ebenso wie auch jenes der politischen Abhängigkeit entspringen beide der gleichen Überlegung, dass die individuelle Bedürfnisbefriedigung um so eher gewährleistet ist, je weniger Distanz herrscht zwischen Bürgern und der (Verwaltungs-) Stelle, die ein bestimmtes öffentliches Gut bereitstellt. Diese Distanz kann aufgrund fachlicher Expertise oder mangelhafter politischer Kontrollmechanismen und autonomen Handlungsspielräumen bestehen. Der Übergewichtung von fachlichen Kriterien auf Kosten der Berücksichtigung der eigentlichen Nachfrage bei der Herstellung eines öffentlichen Guts soll durch ein 
Aufbrechen der hierarchischen Arbeitsteilung entgegengewirkt werden (Obsorne/ Gaebler 1992: 47). Die Zielorientierung anstelle der Verfahrenskontrolle führt zu anderen Anforderungen an die Beamtinnen und Beamten. Nicht mehr spezialisierte Träger abgesonderter Funktionen werden in Amtsstellen mit ,Missionen“ benötigt, sondern breit einsetzbare Generalisten, die eine sektorale Abstimmung fallweise vornehmen können. Die individuellen Präferenzen werden mit abnehmender Professionalisierung weniger verzerrt durch übergeordnete fachliche Konzepte und deshalb besser befriedigt.

- Das Postulat einer möglichst weitgehend politisierten, sprich nicht ernannten, sondern von der betroffenen Bevölkerung oder deren legitimierten Vertretung gewählten Beamtenschaft entspringt ebenfalls dem Willen, individuelle Bürgerpräferenzen möglichst unmittelbar zu befriedigen (Osborne/Gaebler 1992: 47). Die Lösung der Public Choice-Schule liegt zum einen darin, die Verwaltung möglichst klein zu halten und sie zum andern unter die direktdemokratische Kontrolle zu stellen (Kettl 2000; Lane 2000). Die Anbindung der Verwaltung an demokratische Entscheide, um so neuen Bedürfnissen möglichst schnell begegnen zu können, wird verbindlich, wenn Beamte direkt von der Gunst der Stimmbürgerschaft abhängen, da in diesem Fall bei Nicht-Erfüllen einer Mehrheit der individuellen Bedürfnisse die Abwahl droht. Die politische Abhängigkeit erhöht so nicht allein die demokratische Legitimität einer Verwaltung, sondern steigert auch ihre Leistung im Sinne des Kollektivs als Summe seiner Teile.

\section{Politikkoordination als Maß kollektiver Handlungsfähigkeit und Hypothesen}

Wie einleitend erwähnt, stellt die Politikkoordination eine zentrale Herausforderung urbaner Räume dar. Sie ist entsprechend die abhängige Variable der vorliegenden Untersuchung bzw. - gemäß QCA-Terminologie - das zu erklärende Resultat. Mit der Frage nach der Koordination von Politiken ist seit den 1970er Jahren vor allem Fritz W. Scharpf (1972, 1991, 1992, 1993, 1994, 1997) beschäftigt. Scharpf (1993: 58) versteht Politikkoordination als wohlfahrtstheoretisches Konzept: Es sollen „Formen der Abstimmung" beschrieben werden, „deren wohlfahrtstheoretisches Anspruchsniveau über das durch wechselseitige Antizipation in nicht-kooperativen Spielen erreichbare (...) Gleichgewicht hinausgeht.“ Als wohlfahrtstheoretisches Konzept benötigt die Koordination von Politik einen klaren, normativen Bezug. Der ökonomische Maßstab für Wohlfahrt ist das Pareto-Prinzip, das einer Lösung allokative Effizienz zugesteht, ,wenn keine Änderung mehr denkbar ist, welche den Nutzen mindestens eines Beteiligten erhöhen würde, ohne dass irgend ein anderer deshalb schlechter gestellt werden müsste“ (Scharpf 1992: 15). Der „,bloße Verzicht auf unnötige Nutzenvereitelung“ (Scharpf 1991: 624) ist jedoch für die politikwissenschaftliche Beurteilung unzureichend, da Politik ungleich dem Markt in Besitzstände eingreifen kann und nicht allein an der wohlfahrtstheoretischen Effizienz, sondern ebenso an der Verteilungsgerechtigkeit der gefundenen Lösungen interessiert ist.

Als „,bessere Annäherung an einen politisch relevanten Wohlfahrtsbegriff“ bezeichnet Scharpf (1991: 624) das Kaldor-Kriterium (Kaldor 1939), „das alle Massnahmen positiv 
bewertet, deren Nutzen für die Begünstigten groß genug ist, um daraus auch noch die volle Entschädigung aller durch die Maßnahme Benachteiligten bestreiten zu können.“ Die normative Frage der Distributionsgerechtigkeit ist in einem Kaldor-Optimum besser zu lösen als in Lösungen, die nur Pareto-optimal sind. Im Gegensatz zum Pareto-Optimum bleibt bei Kaldor-optimalen Lösungen Raum für politische Verteilungsprozesse.

Das Koordinationsdilemma nach Scharpf (1994: 28) liegt nun darin, dass Kaldoroptimale Lösungen nur in perfekten Hierarchien oder aber in perfekten Verhandlungen erreicht werden können. Beide Koordinationsformen haben den Nachteil, dass sie ihre Funktionsfähigkeit verlieren, wenn ihre Idealbedingungen nicht vollständig erfüllt sind, und sie deshalb in der Realität nicht vorkommen. Die ,games real actors play“ (Scharpf 1997) finden also abseits dieser Idealtypen statt. Vielmehr finden sich institutionell eingebettete Formen von Selbstkoordination, die als Mischformen zwischen den beiden Idealtypen oszillieren. Für die empirische Beschreibung und die Bewertung dieser Formen von „Verhandlungen im Schatten der Hierarchie“ (Scharpf 1972) werden im Folgenden vier Aspekte untersucht: erstens das Auftreten von Koordination, zweitens die Qualität des Koordinationsprozesses, drittens die Qualität des Koordinationsergebnisses und viertens die tatsächliche Umsetzung der Lösung.

Als erste abhängige Variable wird entsprechend obiger Aufzählung das Auftreten von Koordination gewählt, womit konkret das Abstimmen der Handlungsoptionen der verschiedenen in einen gemeinsamen Problemlösungsprozess involvierten Akteure gemeint ist.

Die zweite abhängige Variable ist die formale Rationalität von Koordinationsprozessen. Während in der positiven Koordination neue Projekte multilateral mit allen Handlungsoptionen aller anderen, betroffenen Stellen abgestimmt werden, beschränkt die negative Koordination sich antizipativ und bilateral alle neuen Projekte auf Optionen, die mit den gegebenen und aktuell nicht zur Disposition stehenden Positionen aller Betroffenen verträglich sind (Scharpf 1993). Abgestützt auf die Argumentation von Scharpf (1993, 1994, 1997), dass eine Verhandlung umso näher am Kaldor-Optimum liegende Resultate erzielt, je mehr Policy-Optionen sie aufeinander abstimmt und je konsensueller sie ist, wird jener Koordinationsprozess als formal rational bezeichnet, der freiwillig, multilateral bzw. positiv koordiniert und handlungsrational ist. Als formal mehrheitlich rational werden Mischformen von Verhandlungsprozessen bezeichnet (z.B. Zwangsverhandlung, positive Koordination und Machtrationalität). Als formal mehrheitlich nicht rational wird jener Prozess bezeichnet, in dem unter Zwang verhandelt wird und der bilateral bzw. negativ koordiniert und machtrational ist. Als formal nicht rational werden einseitige Anordnungsentscheide bezeichnet.

Das Kaldor-Optimum wird als dritte abhängige Variable gewählt. Da es sich um ein rein theoretisches Konstrukt handelt, muss das Maß modifiziert werden. Hierzu wird der Begriff der substanziellen Rationalität eingeführt. Im Folgenden wird ein urbanes Projekt dann als substanziell rational bezeichnet, wenn es sich um eine Annäherung an das Kaldor-Optimum handelt, teilweise substanzielle Rationalität bezeichnet die ledigliche Pareto-Superiorität, d.h. der Nutzen für die Begünstigten reicht nur für eine teilweise, jedoch nicht für die volle Entschädigung der Verlierer, während bei einem Pareto-Optimum oder keiner Koordination von fehlender substanzieller Rationalität gesprochen wird. 
Ein koordiniertes Entscheidungsergebnis ist von wenig praktischem Nutzen, wenn es nicht tatsächlich auch realisiert wird. Für eine Beurteilung von Koordinationsqualität als Mass kollektiver Handlungsfähigkeit muss deshalb auch berücksichtigt werden, ob ein Entscheidungsergebnis die politische Akzeptanz findet, die für eine Umsetzung notwendig ist. Die Umsetzung einer Planung selber, d.h. die bauliche Realisierung eines Projekts, ist deshalb die vierte abhängige Variable.

Auf Basis der beiden im Theorieteil vorgestellten Modelle können damit die folgenden Hypothesen zur Erklärung von Koordinationserfolg formuliert werden, wobei sich die neoprogressiven Annahmen $(n p)$ und die Public Choice-Erwartungen $(p c)$ jeweils direkt widersprechen:

Hlnp: Die Zentralisierung der Verwaltungsführung gewährleistet erfolgreiche Politikkoordination.

H1pc: Dezentrale Verwaltungsführung gewährleistet erfolgreiche Politikkoordination.

H2np: Konsolidierte, urbane Raumstrukturen gewährleisten erfolgreiche Politikkoordination.

H2pc: Fragmentierte, urbane Raumstrukturen gewährleisten erfolgreiche Politikkoordination.

H3np: Gebietseinheiten mit einer stark professionellen und spezialisierten Verwaltung gewährleisten erfolgreiche Politikkoordination.

H3pc: Nicht professionelle Generalistenverwaltung gewährleistet erfolgreiche Politikkoordination.

H4np: Politische Autonomie der Verwaltung gewährleistet erfolgreiche Politikkoordination.

H4pc: Politische Abhängigkeit der Verwaltung gewährleistet erfolgreiche Politikkoordination.

\section{Untersuchungsanlage, Wahl der Fälle, Methode, Operationalisierung und Daten}

Für die empirische Überprüfung der postulierten institutionellen Effekte wird eine so genannte Meta-Analyse von siebzehn bestehenden Fallstudien zur urbanen Raumordnungspolitik in neun westeuropäischen Staaten durchgeführt. Meta-Analysen bezeichnen die quantitative Aufarbeitung bestehender Studien, deren Ergebnisse üblicherweise in numerischer Form vorliegen. Die Idee hinter einer solchen Vorgehensweise ist, dass für die empirische Behandlung einer theoretischen Fragestellung die Auswertung bestehender Studien zu Erkenntnissen führen kann, die über die Resultate und Erfahrungen der einzelnen Studien hinausführen (Wolf 1986; Hunter/Schmidt 1990). Zusätzlich lassen sich bei einer Aggregierung der Ergebnisse mehrerer Untersuchungen methodische Schwächen der einzelnen Studien eliminieren und Ergebnisse von größerer Glaubwürdigkeit erzielen. Insbesondere können Fehlerquellen, die aufgrund einer man- 
gelnden Stichprobengrösse der einzelnen Studien entstehen, zum Teil vermindert werden (Glass et al. 1994). In der vorgestellten Untersuchung wird eine quantitative Auseinandersetzung mit qualitativen Einzelfallanalysen durchgeführt, da die behandelten Fragen Wirkungsweisen betreffen, die sich nur durch die diachrone Analyse gesamter Prozessverläufe analysieren lassen. Eine sehr ähnliche Forschungsanlage verwenden Nijkamp et al. (2002), die für ihre Meta-Analyse von urbanen Revitalisierungsprojekten in den Niederlanden ebenfalls auf ex post kodierte, qualitative Fallstudien aufbauen.

Ein zentrales Problem von Meta-Analysen ist die Validität, die durch die Heterogenität der einbezogenen Fälle beeinträchtigt werden kann. Diesem Problem wird in der vorliegenden Untersuchungsanlage auf zwei Arten begegnet: Erstens durch eine begründete Fallwahl mit klaren Kriterien und zweitens durch die einheitliche Kodierung der qualitativen Fallstudien entlang der nachfolgend präsentierten Operationalisierung (Tabellen 2 und 3). Die Grundgesamtheit der vorliegenden Untersuchung bestand aus allen zum Zeitpunkt der Untersuchung greifbaren Studien, die sich sozialwissenschaftlich mit der Frage der Koordination von raumwirksamen Politikfeldern im urbanen Raum befassten. Um in die Untersuchungsgesamtheit aufgenommen zu werden, musste eine Studie vier Kriterien erfüllen: Sie musste eine westeuropäische Metropolitanregion analysieren; sie musste einen Entscheidungsfall zur Koordination von Raum- und Verkehrspolitik untersuchen; sie musste eine Kodierung entlang der präsentierten Operationalisierung zulassen; und sie musste bestimmten formalen wissenschaftlichen Kriterien genügen. Diese Kriterien für die Fallwahl werden im Folgenden kurz dargelegt:

Erstens mussten die Studien Fälle aus urbanen Räumen Westeuropas zum Gegenstand haben. Die Beschränkung auf den westeuropäischen Raum erlaubt die für die Analyse notwendige Streuung der institutionellen Variablen aufgrund unterschiedlicher nationaler Verwaltungs- und Staatskulturen (Knill 1999; Lefèvre 1998; Raphael 2000), während sie gleichzeitig einen spezifischen und gegenüber anderen Weltregionen abgrenzbaren historischen und kulturellen Rahmen (Caramani 2005; Kaelble 2002) vorgibt. Dadurch werden verschiedene kulturelle, historische und politsystemische Variablen kontrolliert und eine grundsätzliche Vergleichbarkeit der Fälle wird gewährleistet.

Zweitens mussten die Fälle dieselben Politikfelder betreffen, um Verzerrungen aufgrund von politikfeldspezifischen Besonderheiten vorzubeugen. Das Problem der Zersiedelung urbaner Räume ist primär ein Problem der wachsenden sozialen und geografischen Mobilität, indem Stadtbewohner bei zunehmendem Wohlstand in die Vorstädte ziehen. Im Zuge der Verlegung des Wohnortes aus der Zentrumsgemeinde wird mehrheitlich der Arbeitsplatz beibehalten, was den Arbeitsweg verlängert. Da die ErschlieBung durch den öffentlichen Verkehr in den weniger dicht besiedelten Agglomerationen schwieriger ist als in den dicht besiedelten Kernstädten, steigt das Volumen des motorisierten Individualverkehrs auf den Straßen, was wiederum primär die Lebensqualität der Kernstädte trifft und entsprechend die Attraktivität der Vororte steigert. Die theoretische Lösung für die städtischen Räume liegt in einer langfristigen Reurbanisierung, d.h. in der umwelt-, sozial- und wirtschaftsverträglichen Umkehrung des Zersiedelungstrends. Den beiden raumwirksamen Politikfeldern Raumplanung und Verkehrsplanung kommt bei dieser vielgestaltigen Aufgabe eine zentrale Rolle zu, indem die Zersiedelung zuerst ein Raumproblem darstellt. Konkret gilt es, Wohnraum sowie Gewerbe- und Dienstleistungsbetriebe an Bahnhöfen und weiteren Knotenpunkten des öffentlichen Verkehrs so- 
wie an geeigneten Punkten des Straßennetzes anzusiedeln, was die grenzüberschreitende Koordination von Raum- und Verkehrspolitik impliziert (Dijst und Schenkel 2002; Holz-Rau und Jansen 2007; Janssen-Jansen 2005; Kaufmann und Sager 2006, Sager 2002). Die Raum- und die Verkehrsplanung können deshalb als geeignete Testfälle für die Untersuchung von Politikkoordination betrachtet werden.

Drittens mussten die Fallstudien eine Analyse oder zumindest die Beschreibung der institutionellen Variablen beinhalten, die in dieser Untersuchung im Vordergrund stehen, und eine Bewertung des Koordinationsprozesses beziehungsweise seines Ergebnisses vornehmen.

Viertens schließlich fanden die formalen Kriterien von Wolf (1993: 166) für die Wahl von Fallstudien Anwendung. Eine Fallstudie musste, um berücksichtigt zu werden, auf empirischen Daten und nicht auf Modellrechnungen basieren, einen mindestens fünfzehnseitigen Analyseteil umfassen, um eine argumentierte Kodierung zuzulassen, eine Bibliografie und Quellenangaben beinhalten und durfte nicht von am untersuchten Fall beteiligten Personen verfasst worden sein.

Das Vorgehen bei der Fallauswahl bestand aus einem breiten Literaturstudium, wobei einerseits publizierte Studien, andererseits über Forschungsverbünde auch graue Literatur gesichtet wurde. Diese Suche führte zu insgesamt siebzehn Fallstudien, die auf Basis dieser Kriterien in die Analyse einbezogen wurden (vgl. Tabelle 1). ${ }^{2}$ Im Zentrum der Auswahlkriterien stand die Eignung des untersuchten Problems für die Fragestel-

Tabelle 1: Die ausgewählten Fallstudien

\begin{tabular}{|c|c|c|}
\hline Fallstudie & Land & Quelle \\
\hline 1. Mobilitätsvertrag in Flandern & Belgien & Steenberghen 2000 \\
\hline $\begin{array}{l}\text { 2. Öresund-Verbindung von Kopenhagen } \\
\text { und Malmö }\end{array}$ & Dänemark/Schweden & Matthiessen 2000 \\
\hline 3. Kopenhagen Metro & Dänemark & Matthiessen 2000 \\
\hline 4. Verkehrskonzept Freiburg & Deutschland & Bratzel 1999 \\
\hline $\begin{array}{l}\text { 5. Agglomerationsverkehrsplanung } \\
\text { Marseille }\end{array}$ & Frankreich & Margail and Offner 2000 \\
\hline $\begin{array}{l}\text { 6. Agglomerationsverkehrsplanung } \\
\text { Toulouse }\end{array}$ & Frankreich & Margail and Offner 2000 \\
\hline 7. Agglomerationsverkehrsplanung Orléans & Frankreich & Margail and Offner 2000 \\
\hline 8. Stadtentwicklung Newcastle & Großbritannien & Guy et al. 2000 \\
\hline 9. Planungsvereinbarung in Saronno & Italien & Morandi et al. 2000 \\
\hline 10. Integrierte Stadtplanung in Rom & Italien & Sessa and Gualdi 2000 \\
\hline $\begin{array}{l}\text { 11. Entwicklungsplan für die Agglomeration } \\
\text { Groningen }\end{array}$ & Niederlande & Voogd 2000 \\
\hline 12. Verkehrskonzept Amsterdam & Niederlande & Bratzel 1999 \\
\hline 13. Stadttunnel in Malmö & Schweden & Book and Eskilsson 2000 \\
\hline $\begin{array}{l}\text { 14. Erneuerung des Stadtzentrums von } \\
\text { Staffanstorp }\end{array}$ & Schweden & Book and Eskilsson 2000 \\
\hline $\begin{array}{l}\text { 15. Korrektur des Llobregat-Flusses in } \\
\text { Barcelona }\end{array}$ & Spanien & Junyent 2000 \\
\hline 16. Hafenausbau Barcelona & Spanien & Junyent 2000 \\
\hline 17. Flughafenausbau Barcelona & Spanien & Junyent 2000 \\
\hline
\end{tabular}

2 Für die Präsentation aller berücksichtigten Fälle siehe Sager (2002: 254-280). 
lung und nicht die Fälle selber. Das heisst, die Fallstudien mussten vergleichbar sein, nicht die darin untersuchten Fälle. Diese sind entsprechend nicht homogen. Es finden sich dabei sowohl Studien zu Großregionen wie Barcelona oder Marseille als auch Untersuchungen von kleineren urbanen Räumen wie Groningen oder Freiburg. Ebenfalls besteht keine gleichmässige Verteilung auf die rahmengebenden europäischen Staaten. Während die Vergleichbarkeit der Studien also durch die Auswahlkriterien soweit möglich abgesichert wurde, kann diese Auswahl naheliegenderweise keinen Anspruch auf Vollständigkeit oder Repräsentativität erheben. Diesem Umstand wird jedoch Rechnung getragen mit der Wahl der Methode, die nachfolgend präsentiert wird.

Diese siebzehn Fallstudien werden im Folgenden systematisch verglichen. Die vier Postulate pro zu testendes Modell können als kohärente Gruppen von interdependenten institutionellen Einflussfaktoren verstanden werden. Der empirische Teil legt denn auch den Schwerpunkt auf die kombinierten Effekte der institutionellen Charakteristika. Hierzu wird auf die Methode der Qualitative Comparative Analysis (QCA) zurückgegriffen (De Meur/Rihoux 2002; Ragin 1987, 2000; Wagschal 1999: 291), die besonders geeignet ist für den Vergleich einer mittleren Anzahl Fälle. QCA basiert auf den Grundlagen der Booleschen Algebra, welche mit dem Binärsystem operiert. Eine Voraussetzung für die Anwendung von QCA ist das Vorliegen ausschließlich binär kodierter Variablen. Mit der Dichotomisierung geht ein Informationsverlust einher. Gleichwohl wurde auf eine feiner abgestufte Kodierung, die mit der Weiterentwicklung der QCA, den so genannten Fuzzy Sets (fsQCA), ausgewertet hätte werden können, verzichtet, da damit die Gefahr von arbiträren Kodierungen zugenommen hätte, zumal es sich bei den Fällen um qualitative Fallstudien unterschiedlicher Autorenschaft handelt. Mit der nachfolgend vorgestellten Operationalisierung wurden klare qualitative Schwellenwerte zur Abgrenzung der Präsenz eines Merkmals (Code 1) von dessen Absenz (Code 0) angestrebt. Auf diese Weise wurden die Fälle einheitlich entlang der Operationalisierung von Politikkoordination (Tabelle 2) sowie jener der vier Organisationsdimensionen (Tabelle 3) kodiert.

Tabelle 2 zeigt die Operationalisierung der vier abhängigen Variablen.

Die vier beschriebenen Organisationsdimensionen können für die vorliegende Untersuchung als unabhängige Variablen bzw. Bedingungen operationalisiert werden, in welchen die beiden Schulen jeweils die unterschiedlichen Ausprägungen aufweisen. Tabelle 3 präsentiert die Operationalisierung der unabhängigen Variablen.

Die Werte, die die Variablen annehmen, sind teils verzerrt, da die Fälle nicht auf die europäischen Länder gleichverteilt und die Variablen teils vom Verfassungskontext abhängig sind. Diesem Umstand begegnet QCA durch die Logik der Booleschen Algebra. Die Boolesche Addition funktioniert mit dem logischen Operator ,oder', d.h., wenn zwei Variablen A und B eine 1 ausweisen und das Ergebnis $Z$ ist ebenfalls 1, dann gilt der Zusammenhang $\mathrm{A}+\mathrm{B}=\mathrm{Z}$, was mit den eingesetzten Werten die Gleichung $1+1=1$ ergibt. Ebenfalls ist das Ergebnis $Z=1$, wenn nur A oder nur B den Wert 1 haben, also gilt: $0+1=1$ und $1+0=1$. Die Boolesche Multiplikation wendet im Gegensatz zur Addition den logischen Operator ,und' an. Die Idee von QCA ist, für die gefundenen Ausprägungen der untersuchten Bedingungen so genannte Wahrheitstafeln zu erstellen, in denen die beobachteten Kombinationen von Faktoren zusammengetragen werden. Die Konfigurationen werden anschließend in additive und multiplikative Formeln über- 
Tabelle 2: Operationalisierung der abhängigen Variablen/Resultate

\begin{tabular}{|c|c|c|c|c|}
\hline Resultat & gegeben & $\begin{array}{l}\text { Mehrheitlich } \\
\text { gegeben }\end{array}$ & $\begin{array}{l}\text { Mehrheitlich } \\
\text { nicht gegeben }\end{array}$ & Nicht gegeben \\
\hline & \multicolumn{2}{|l|}{ Code 1} & \multicolumn{2}{|c|}{ Code 0} \\
\hline $\begin{array}{l}\text { Koordination } \\
\text { Symbol: } K\end{array}$ & $\begin{array}{l}\text { - Eine Anpassung der Akteur- } \\
\text { haltungen findet statt }\end{array}$ & - & - & $\begin{array}{l}\text { - Eine An- } \\
\text { passung der } \\
\text { Akteurhal- } \\
\text { tungen findet } \\
\text { nicht statt }\end{array}$ \\
\hline $\begin{array}{l}\text { Formelle } \\
\text { Rationalität } \\
\text { Symbol: } F\end{array}$ & $\begin{array}{l}\text { - Koordination ist freiwillig } \\
\text { - Koordination ist positiv, d.h. } \\
\text { multilateral } \\
\text { - Verantwortungsethik dominiert }\end{array}$ & $\begin{array}{l}\text { - Koordination ist } \\
\text { verhandelt }\end{array}$ & $\begin{array}{l}\text { - Koordination ist } \\
\text { auferlegt und } \\
\text { nicht verhandelt }\end{array}$ & $\begin{array}{l}\cdot \text { Keine } \\
\text { Koordination }\end{array}$ \\
\hline $\begin{array}{l}\text { Substanzielle } \\
\text { Rationalität } \\
\text { Symbol: } S\end{array}$ & $\begin{array}{l}\text { - Die gefundene Lösung } \\
\text { befriedigt die Interessen aller } \\
\text { Betroffenen, d.h. der Nutzen } \\
\text { der Gewinner reicht für die } \\
\text { Kompensation der Verlierer }\end{array}$ & $\begin{array}{l}\text { - Die Interessen- } \\
\text { befriedigung der } \\
\text { Gewinner reicht } \\
\text { für die teilweise } \\
\text { Kompensation der } \\
\text { Verlierer }\end{array}$ & - & $\begin{array}{l}\text { - Die Interes- } \\
\text { senbefrie- } \\
\text { digung der } \\
\text { Gewinner } \\
\text { bedeutet einen } \\
\text { Verlust für die } \\
\text { Verlierer }\end{array}$ \\
\hline $\begin{array}{l}\text { Umsetzung } \\
\text { Symbol: } U\end{array}$ & $\begin{array}{l}\text { - Die gefundene Lösung wird } \\
\text { umgesetzt }\end{array}$ & $\begin{array}{l}\text { - Die gefundene } \\
\text { Lösung wird } \\
\text { mehrheitlich } \\
\text { umgesetzt }\end{array}$ & $\begin{array}{l}\text { - Die gefundene } \\
\text { Lösung wird } \\
\text { mehrheitlich } \\
\text { nicht umgesetzt }\end{array}$ & $\begin{array}{l}\text { - Die gefun- } \\
\text { dene Lösung } \\
\text { wird nicht } \\
\text { umgesetzt }\end{array}$ \\
\hline
\end{tabular}

Tabelle 3: Operationalisierung der unabhängigen Variablen/Bedingungen

\begin{tabular}{|c|c|c|}
\hline Bedingung & Neoprogressives Modell & Public Choice Modell \\
\hline & Code 1 & Code 0 \\
\hline Zentralisierungsgrad & $\begin{array}{l}\text { - Hierarchische Projektstruktur } \\
\text { - In der Hierarchie der zuständigen } \\
\text { Amtsstelle hoch angesiedelte } \\
\text { Projektleitung } \\
\text { - Position des primus inter pares mit } \\
\text { zentralen Koordinationsaufgaben } \\
\text { im zuständigen Koordinationsorgan } \\
\text { innerhalb der Verwaltung }\end{array}$ & $\begin{array}{l}\text { - Flache Projektstrukturen } \\
\text { - In der Hierarchie der zustän- } \\
\text { digen Amtsstelle tief angesiedelte } \\
\text { Projektleitung } \\
\text { - Das zuständige Koordinationsorgan } \\
\text { innerhalb der Verwaltung funktioniert } \\
\text { als Ort des freien Informationsaus- } \\
\text { tausches ohne zentrale Schaltstelle }\end{array}$ \\
\hline Konsolidierungsgrad & $\begin{array}{l}\text { - Status der Agglomeration als poli- } \\
\text { tische Einheit bzw. eigene föderale } \\
\text { Ebene (Region, Bezirk) }\end{array}$ & $\begin{array}{l}\text { - Kein Status der Agglomeration } \\
\text { als politische Einheit: Kern- } \\
\text { stadt mit gleichberechtigten } \\
\text { Agglomerationsgemeinden }\end{array}$ \\
\hline $\begin{array}{l}\text { Professionalisierungs- } \\
\text { grad }\end{array}$ & $\begin{array}{l}\text { - Kein Einfluss der nicht-pro- } \\
\text { fessionellen Verwaltungen der } \\
\text { suburbanen Gemeinden im } \\
\text { Entscheidungsprozess } \\
\text { - Aufgaben, die professionelle Ex- } \\
\text { pertise verlangen, werden vorrangig } \\
\text { von der Verwaltung übernommen }\end{array}$ & $\begin{array}{l}\text { - Die nicht-professionellen Ver- } \\
\text { waltungen der suburbanen Ge- } \\
\text { meinden haben Einfluss im } \\
\text { Entscheidungsprozess } \\
\text { - Aufgaben, die professionelle Ex- } \\
\text { pertise verlangen, werden vorrangig } \\
\text { ausgelagert }\end{array}$ \\
\hline $\begin{array}{l}\text { Grad an politischer } \\
\text { Autonomie }\end{array}$ & $\begin{array}{l}\text { - Die politische Sphäre ist von der } \\
\text { technischen Sphäre getrennt }\end{array}$ & $\begin{array}{l}\text { - Die politische Sphäre und die tech- } \\
\text { nische Sphäre sind nicht klar getrennt }\end{array}$ \\
\hline
\end{tabular}




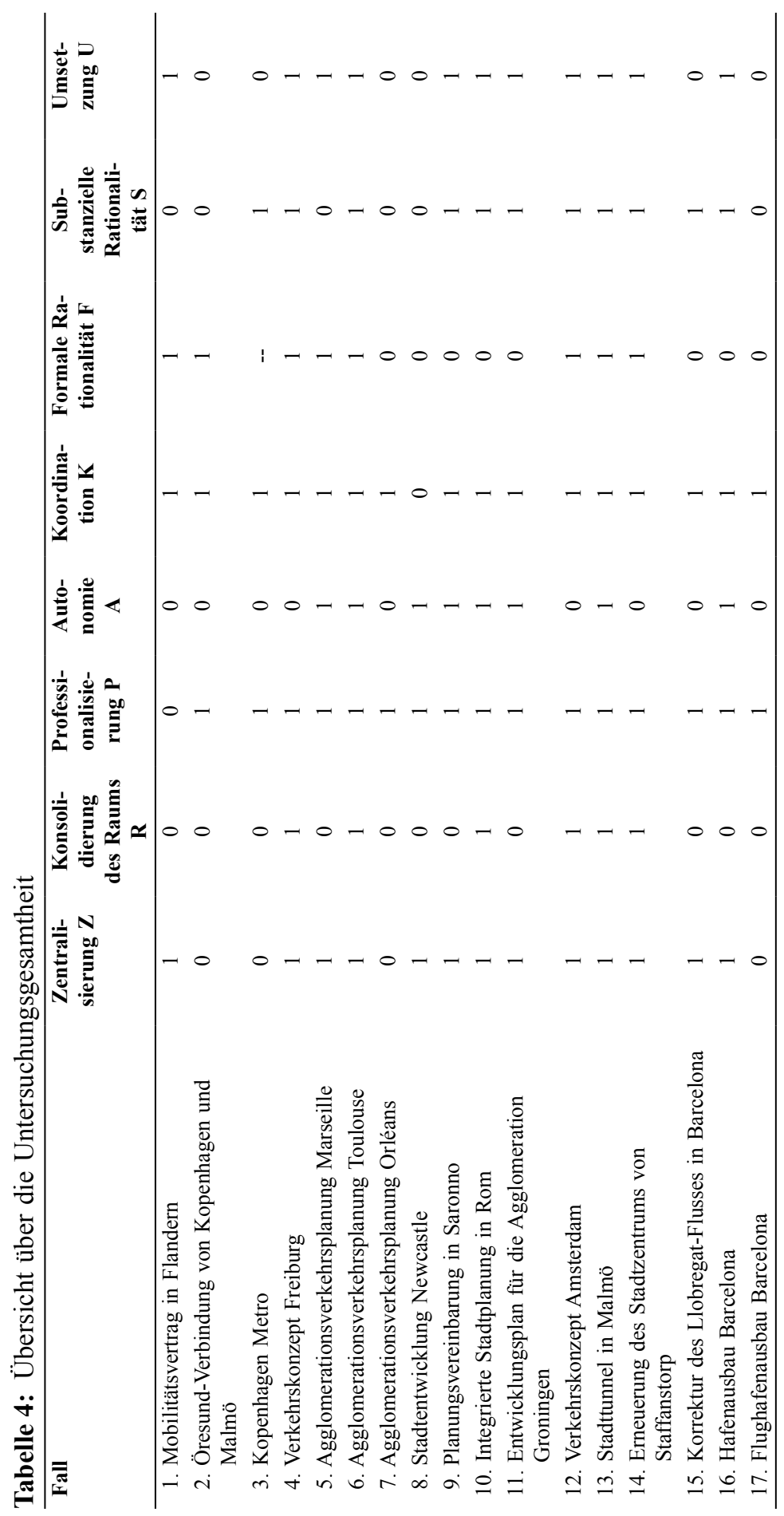


tragen, wobei der Wert 1 durch Großbuchstaben, der Wert 0 durch Kleinbuchstaben gekennzeichnet wird.

Die Fallzahl zu jeder beobachteten Ursachenkonfiguration ist dabei zweitrangig, weshalb die genannten Ungleichverteilungen der Fälle kein Problem darstellen. Nicht berücksichtigt werden Fälle mit fehlenden Daten. Mittels eines Minimierungsalgorithmus (Quine-McCluskey-Minimierungsalgorithmus) werden anschließend in zwei Schritten die überflüssigen Variablen aus den formalisierten Daten der Wahrheitswertetafeln eliminiert, um so zu einer Erklärung der abhängigen Variablen zu gelangen, die mehrere Einflussfaktoren in spezifischen Kombinationen beinhaltet. Diese Analyse wird in den folgenden Abschnitten für jede der vier abhängigen Größen durchgeführt.

Tabelle 4 gibt eine Übersicht über die aus der binären Kodierung der siebzehn Testfälle gewonnenen Daten.

\section{Resultate}

Die Ergebnisse der Analyse mit QCA werden entlang der vier abhängigen Variablen präsentiert.

\section{Institutionelle Konfigurationen für das Zustandekommen von Koordination}

Von sechzehn möglichen Kombinationsmöglichkeiten $\left(4^{2}\right)$ zeigen sich in der Untersuchungsgesamtheit sechs Konstellationen der vier Bedingungen für die abhängige Grösse ,Koordination“. Eine Konstellation findet sich mit beiden Resultaten: Die Kombination ZrPA führte in vier der untersuchten Fallstudien (Fälle 5, 9, 11 und 16) zu Koordination, in einer Studie (Fall 8) dagegen nicht. Für die Behandlung solcher widersprüchlicher Fälle sieht Ragin (1987: 116) mehrere Möglichkeiten vor, von denen hier jene als sinnvoll erachtet wird, die vorsieht, das Outcome zweideutiger Kombinationen mit $0 \mathrm{zu}$ kodieren und nur jene Konstellationen in die Analyse für ein Outcome 1 einzubeziehen, die eindeutig sind. ${ }^{3}$ Die Informationen aus den kodierten Fallstudien können also in folgende Formel übertragen werden:

$\mathrm{K}=\mathrm{zrPa}+\mathrm{Zrpa}+\mathrm{ZrPa}+\mathrm{ZRPa}+\mathrm{ZRPA}$

Dieses Ergebnis wird nun in einem schrittweisen Verfahren auf die Kernvariablen reduziert. Die nicht weiter zu minimierende Kernlösung lautet:

$\mathrm{K}=\mathrm{Zra}+\mathrm{ZRP}+\mathrm{rPa}$

3 Die zweite Möglichkeit ist die Umkodierung in eine nicht existierende Kombination, ein Vorgehen, das hier als arbiträr verworfen wird. Die dritte Möglichkeit ist die einheitliche Kodierung des Outcomes mit 1, was zu mehr Lösungen führt, aber problematisch zu interpretieren ist (Ragin 1987: 116). 
Das Ergebnis der QCA lautet entsprechend: Ein Koordinationsprozess findet dann statt, wenn

a) eine zentralisierte Organisation und eine fragmentierte Raumordnung und eine geringe Autonomie der Verwaltung vorliegen oder

b) eine zentralisierte Organisation und eine konsolidierte Raumstruktur und eine hohe Professionalisierung vorliegen oder

c) eine fragmentierte Raumstruktur und eine hohe Professionalisierung und eine geringe Autonomie der Verwaltung vorliegen.

Das ausformulierte Ergebnis zeigt, dass keine der untersuchten vier Variablen eine notwendige noch eine hinreichende Bedingung für das Zustandekommen von Koordination darstellt, indem keiner der Faktoren in allen Ursachenkonstellationen vorkommt noch alleine zum Resultat ,Koordination“ führt. Das Ergebnis spricht weder für das neoprogressive noch für das Public Choice-Modell. Vielmehr hängt es von der jeweiligen Faktorenkonfiguration $\mathrm{ab}$, in welcher Form sich die Bedingungen positiv auf das Auftreten von Koordination auswirken.

\section{Institutionelle Konfigurationen für Formale Rationalität}

Für die abhängige Größe Formale Rationalität (F) finden sich sechs Konfigurationen, von denen drei widersprüchlich sind und deshalb eliminiert werden. Von den drei eindeutigen Konfigurationen führen zwei zu formal rationalen Koordinationsverhandlungen (F). Verordnete Koordination (f) ist dagegen das Ergebnis von nur einer eindeutigen Konfiguration. Die Daten können in folgende Gleichung übertragen werden:

$\mathrm{F}=\mathrm{Zrpa}+\mathrm{ZRPa}$

Aus dieser Gleichung können keine Implikanten gebildet werden. Die Gleichung kann aber umgeschrieben werden in:

$\mathrm{F}=\mathrm{Za}(\mathrm{rp}+\mathrm{RP})$

Formal rationale Koordination kann also in jenen Fällen beobachtet werden, in denen eine zentralisierte Organisationsstruktur und eine geringe Autonomie der Verwaltung vorliegen und

a) entweder eine fragmentierte Raumordnung und eine geringe Professionalisierung oder

b) eine konsolidierte Raumordnung und eine hohe Professionalisierung vorliegen.

Die Merkmalsausprägungen ,hoher Zentralisierungsgrad“ und ,niedriger Autonomiegrad" stellen somit notwendige Bedingungen dar, sind aber nicht hinreichend, da sie nur in Kombination mit den übrigen beiden Variablen $\mathrm{zu}$ formal rationalen Koordinationsverhandlungen führen. Die übrigen beiden Bedingungen weisen weder notwendige noch hinreichende Ausprägungen auf. 
Konsolidierte Raumstrukturen haben dann einen positiven Einfluss auf formale rationale Koordination, wenn gleichzeitig eine hohe Zentralisierung des institutionellen Rahmens, eine hohe Professionalisierung und eine geringe Autonomie der Verwaltung bestehen. Demgegenüber findet auch in fragmentierten Strukturen formal rationale Koordination statt, hier aber dann, wenn gleichzeitig eine tiefe Professionalisierung zu beobachten ist. In beiden Fällen spielt die horizontale Integration des institutionellen Rahmens eine vordringliche Rolle. Zentralisierung unterstützt formale Rationalität oder kann sie gar kompensieren. Ein weiteres Ergebnis zeigt, dass eine politisch abhängige Verwaltung eher formal rationale Koordinationsverhandlungen initiiert als eine apolitische. In eher technokratisch organisierten politischen Systemen Westeuropas mit sehr starken urbanen Verwaltungen erhöht die bessere Berücksichtigung politischer Interessen damit offensichtlich die Prozessqualität von Politikkoordination.

\section{Institutionelle Konfigurationen für das Zustandekommen von substanzieller Rationalität}

Die Fallstudien enthalten sechs unterschiedliche Konfigurationen für die abhängige Größe Substanzielle Rationalität (S), wovon zwei widersprüchlich sind und eine zu einem negativen Ergebnis führt. Drei Konfigurationen führen dagegen zu substanzieller Rationalität, die Ausgangsgleichung lautet:

$\mathrm{S}=\mathrm{ZrPa}+\mathrm{ZRPa}+\mathrm{ZRPA}$

Aus dieser Gleichung können zwei Implikanten gebildet werden. Die minimierte Gleichung lautet:

$\mathrm{S}=\mathrm{ZPa}+\mathrm{ZRP}$

Die Gleichung kann wiederum umgeschrieben werden in:

$\mathrm{S}=\mathrm{ZP}(\mathrm{a}+\mathrm{R})$

Substanzielle Rationalität wird also dann erreicht, wenn eine zentralisierte Organisationsstruktur und eine hohe Professionalisierung vorliegen und

a) entweder eine geringe Autonomie der Verwaltung oder

b) eine konsolidierte Raumordnung vorliegt.

Es zeigt sich, dass für die Erreichung von substanziell rationalen Lösungen zwei Merkmalsausprägungen notwendig sind, nämlich eine hohe Zentralisierung und eine hohe Professionalisierung, während die beiden übrigen Variablen nicht notwendigerweise auftreten müssen. Keine der Variablen bietet eine hinreichende Ursache für substanzielle Rationalität.

Mit Ausnahme des Effektes der politischen Anbindung von Verwaltung entsprechen die Resultate den neoprogressiven Postulaten. Wie bei der formalen Rationalität kann der positive Einfluss geringer Autonomie mit den generell eher geringen Partizipations- 
möglichkeiten in den europäischen Städten erklärt werden. Es muss bei der Gegenüberstellung von Professionalisierung und Autonomie aber hervorgehoben werden, dass erstere eine notwendige und damit wichtigere Voraussetzung für substanziell rationale Lösungen ist, während letztere eine austauschbare Bedingung und damit zweitrangig ist.

\section{Institutionelle Konfigurationen für Umsetzung}

Für die abhängige Grösse Umsetzung finden sich sechs Konfigurationen, wovon eine widersprüchlich ist und zwei zu einem negativen Outcome führen. In die QCA werden also drei Konfigurationen einbezogen, die in die folgende Gleichung überführt werden können:

$\mathrm{U}=\mathrm{Zrpa}+\mathrm{ZRPa}+\mathrm{ZRPA}$

Indem ZRPa und ZRPA zu ZRP zusammengeführt werden, kann die Gleichung wie folgt minimiert werden:

$\mathrm{U}=\mathrm{Zrpa}+\mathrm{ZRP}$

Da diese Lösung nicht mehr weiter reduziert werden kann, folgt aus der QCA, dass eine Realisierung von Planungen dort stattfindet, wo

a) eine zentralisierte Organisation und eine fragmentierte Raumordnung und eine tiefe Professionalisierung und eine tiefe Autonomie der Verwaltung vorliegen oder

b) eine zentralisierte Organisation und eine konsolidierte Raumstruktur und eine hohe Professionalisierung vorliegen.

Die Lösung von QCA ist von besonderem Interesse, da sie bei zwei entgegen gesetzten Konstellationen dasselbe Resultat bestimmt, abhängig davon, ob eine zusätzliche Merkmalsausprägung auftritt oder nicht. Konkret findet bei Auftreten der notwendigen Bedingung, hohe Zentralisierung' sowohl bei fragmentierten wie bei konsolidierten Raumstrukturen und sowohl bei hoher wie bei tiefer Professionalisierung eine Umsetzung statt, allein abhängig davon, ob die Verwaltung politisch abhängig ist oder nicht.

In Ergänzung zu den bisherigen Erkenntnissen, die mehrheitlich für die neoprogressiven Annahmen sprechen, zeigt sich also, dass auch unter diesen Postulaten entgegengesetzten Bedingungen eine Umsetzung stattfindet, diese aber sehr viel stärker politisiert ausfällt als in konsolidierten Räumen mit hoch professionalisierten Strukturen. Die Interpretation ist damit zulässig, dass in konsolidierten Räumen Verbesserungen der räumlichen Situation durch primär technisch orientierte Expertenlösungen, in fragmentierten Räumen dagegen durch politisch dominierte und nicht allein technisch legitimierte Lösungen gefunden werden. 
Tabelle 5: Wahrheitswertetafel für die abhängige Größe Implementation I

\begin{tabular}{cccccc}
\hline $\begin{array}{c}\text { Zentralisie- } \\
\text { rung Z }\end{array}$ & $\begin{array}{c}\text { Konsolidie- } \\
\text { rung des } \\
\text { Raums R }\end{array}$ & $\begin{array}{c}\text { Professionali- } \\
\text { sierung P }\end{array}$ & Autonomie A & $\begin{array}{c}\text { Implementa- } \\
\text { tion I }\end{array}$ & Fallzahl N \\
\hline 0 & 0 & 1 & 0 & 0 & 1 \\
1 & 0 & 1 & 0 & 0 & 1 \\
1 & 0 & 1 & 1 & 1 & 3 \\
1 & 1 & 1 & 0 & 1 & 3 \\
1 & 1 & 1 & 1 & 1 & 3 \\
\hline
\end{tabular}

Wann werden substanziell rationale Lösungen umgesetzt?

Nach der getrennten Diskussion jeder abhängigen Größe soll in diesem Abschnitt nun auch noch die Frage angegangen werden, unter welchen Bedingungen substanziell rationale Lösungen umgesetzt werden. Für die Behandlung dieser Frage wurde der Datensatz mit der neuen Variable Implementation I ergänzt, die den Wert 1 annimmt, wenn eine substanziell rationale Lösung umgesetzt wird, und die den Wert 0 annimmt, wenn eine substanziell rationale Lösung nicht umgesetzt wird. Elf Fälle weisen eine der beiden Ausprägungen der Variable auf und können ausgewertet werden. Die Wahrheitswertetafel (Tabelle 5) weist fünf Ursachenkonfigurationen auf, von denen alle eindeutig sind. Zwei Konfigurationen führen zu keiner Umsetzung, drei führen zu einer Umsetzung. In einem ersten Schritt können jene Konfigurationen, die zu einer Umsetzung führen, in eine Gleichung übertragen werden:

$\mathrm{I}=\mathrm{ZrPA}+\mathrm{ZRPa}+\mathrm{ZRPA}$

Die reduzierte und nicht weiter zu minimierende Formel lautet:

$\mathrm{I}=\mathrm{ZPA}+\mathrm{ZRP}$

Diese Lösung stützt eindeutig das neoprogressive Modell in der Frage, wann substanziell rationale Lösungen umgesetzt werden. Eine Realisierung findet dann statt, wenn ein hoher Zentralisierungsgrad der Organisation und eine starke Professionalisierung und

a) eine hohe Autonomie der technischen Verwaltung oder

b) ein hoher Konsolidierungsgrad vorliegen.

In einem zweiten Schritt ist nun zu fragen, wann eine substanziell rationale Lösung gerade nicht realisiert wird. Die Wahrheitswertetafel enthält folgende beiden Konfigurationen:

$\mathrm{i}=\mathrm{zrPa}+\mathrm{ZrPa}$

Die beiden Konfigurationen können zum Implikanten rPa verschmolzen werden, da die Ausprägung von Z keinen Unterschied für das Outcome macht. Es folgt: 
$\mathrm{i}=\mathrm{rPa}$

Die Lösung von QCA hält also fest, dass die Kombination von fragmentierter Raumstruktur und hohem Professionalisierungsgrad und einer geringen Autonomie der Verwaltung sowohl eine notwendige wie eine hinreichende Bedingung für das Ausbleiben der Realisierung einer substanziell rationalen Planung darstellt.

Beide Ergebnisse weisen das neoprogressive Modell als erfolgreicher hinsichtlich der Realisierung koordinierter Politiklösungen aus. Während beim positiven Ergebnis sowohl ein hoher Zentralisierungsgrad wie ein starkes Expertenwissen in der Verwaltung als notwendige Bedingungen, die in Kombination mit konsolidierten Raumstrukturen oder aber einer hohen Verwaltungsautonomie wirken - alles neoprogressive Postulate -, erweist sich jene Konfiguration, die eine Umsetzung verhindert, als besonders effektive gegenseitige Blockade von neoprogressiven und Public Choice-Postulaten. Von besonderem Interesse ist, dass diese Konfiguration rPa vorgängig in Gleichung (1.2) als eine mögliche Ursachenkonstellation für das Zustandekommen von Koordination identifiziert wurde. Koordinationsprozessen ist also nicht allein aufgrund ihres Auftretens Erfolg hinsichtlich Entscheidung und Umsetzung gesichert. Vielmehr kann dieselbe Ursachenkonfiguration auf der einen Seite zu Koordinationsprozessen führen und auf der anderen Seite die Realisierung von substanziell rationalen Lösungen verhindern.

$\mathrm{Da}$ in keinem Fall eine Ursachenkonfiguration vorliegt, die eine Kombination von Public Choice-Postulaten als leistungsfähig bei der Umsetzung koordinierter Lösungen ausweisen würde, wird in diesem Vergleich das neoprogressive Modell als bessere Voraussetzung für die Realisierung von substanziell rationalen Planungsentscheiden und damit für die „Herstellung und Stabilisierung kollektiver Handlungsfähigkeit“ (Schimank 2002: 31) bezeichnet.

\section{Schlussfolgerungen}

Der vorliegende Aufsatz fragte nach den institutionellen Bedingungen kollektiver Handlungsfähigkeit im urbanen Raum. Die empirischen Ergebnisse aus dem Vergleich von siebzehn Fallstudien zu raum- und verkehrsplanerischen Entscheidungsprozessen in westeuropäischen Stadträumen weisen abschliessend das neoprogressive Modell als das erfolgreichere der beiden untersuchten Verwaltungsmodelle aus. Dies zeigt sich beispielhaft an der Umsetzung substanziell rationaler Lösungen als Mass für erfolgreich gemeisterte Interdependenzprobleme. In der Analyse erweisen sich ein hoher organisatorischer Zentralisierungsgrad der Projektstrukturen sowie ein hoher Professionalisierungsgrad der beteiligten Akteure als notwendige Bedingungen für die Realisierung von Lösungen, die insofern dem Kaldor-Optimum entsprechen, als der Nutzen für die Begünstigten groß genug ist, um eine Entschädigung aller durch die Massnahme Benachteiligten zu ermöglichen. Zusätzlich müssen eine hohe Autonomie der Verwaltung oder aber ein hoher Konsolidierungsgrad vorliegen. Substanziell rationale Lösungen werden damit eher umgesetzt, wenn sie unter institutionellen Bedingungen erarbeitet worden sind, die den Postulaten der Vertreter der neoprogressiven Schule entsprechen. 
Die in der QCA festgestellte Komplexität institutioneller Wechselwirkungen legt hinsichtlich institutioneller Reformen in Metropolitanräumen allerdings keine umfassenden institutionellen Fertiglösungen nahe, die mit dem ,goldenen Schnitt' zu einer Totalreform bestehender organisationeller Arrangements führen, sondern das induktive Vorgehen in einzelnen Schritten für konkrete Probleme. Gleichzeitig sprechen die Resultate für eine gewisse Rehabilitierung der klassischen Verwaltung im Sinne einer vertikal ausdifferenzierten Linienorganisation mit hierarchischen Kontrollmechanismen. Wie die Testfälle gezeigt haben, können durch hierarchische Entscheide Koordinationskosten gespart und Konflikten vorgebeugt werden. Gesamtwohlfahrtssteigernde Lösungen werden so mit geringerem Aufwand erreicht und weisen eine höhere politische Akzeptanz auf.

Diese Ausführungen stehen in einem gewissen Gegensatz zum aktuellen verwaltungswissenschaftlichen Mainstream, der für eine möglichst weitgehende Auflösung von Hierarchien und die Schaffung horizontaler Marktsituationen zwischen einzelnen Amtsstellen einsteht. Gerade bei der Frage der Politikkoordination ist jedoch zu beachten, dass die von polit-ökonomischen Reformmodellen wie dem New Public Management propagierten Marktmechanismen weniger kooperatives denn autarkes staatliches Handeln fördern. Betriebswirtschaftliches Effizienzdenken führt in seiner Logik eher zu Wettbewerb als zu Zusammenarbeit und widerspricht damit sowohl der Koordination zwischen Politikfeldern als auch zwischen Gebietseinheiten.

\section{Literatur}

Benz, Arthur. 1994. Kooperative Verwaltung. Funktionen, Voraussetzungen und Folgen. BadenBaden: Nomos.

Berg-Schlosser, Dirk. 1997. Makro-qualitative vergleichende Methoden. In: Berg-Schlosser, Dirk und Ferdinand Müller-Rommel (Hg.). Vergleichende Politikwissenschaft. Opladen: Leske+Budrich. 67-88

Berg-Schlosser, Dirk und Sven Quenter. 1996. Makro-quantitative vs. makro-qualitative Methoden in der Politikwissenschaft - Vorzüge und Mängel komparativer Verfahrensweisen am Beispiel der Sozialstaatstheorie. In: Politische Vierteljahresschrift 1 (37). 100-118.

Blatter, Joachim. 2005. Metropolitan Governance in Deutschland: Normative, utilitaristische, kommunikative und dramaturgische Steuerungsansätze. In: Swiss Political Science Review 1 (11). 119-155.

Book, Karin and Lena Eskilsson. 2000. Co-ordination Between Transport and Land-Use Planning in the Expansive Scania Region. In: COST 332 - Transport and Land-Use Policies: Innovations in Institutional Arrangements for Co-ordination. Final Report of the Action. Brüssel: European Commission. 117-135

Bratzel, Stefan. 1999. Erfolgsbedingungen umweltorientierter Verkehrspolitik in Städten. Analysen zum Policy-Wandel in den, relativen Erfolgsfällen'Amsterdam, Groningen, Zürich und Freiburg (i. Brg.). Basel, Boston, Berlin: Birkhäuser.

Budäus, Dietrich. 1994. Public Management. Konzepte und Verfahren zur Modernisierung öffentlicher Verwaltungen. Berlin: Ed. Sigma.

Caramani, Daniele (Hg.). 2005. Images of Europe: Cultural Diversity and European Integration. London: Routledge.

De Meur, Gisèle et Benoît Rihoux. 2002. L'analyse quali-quantitative comparée (AQQCQCA). Approches, techniques et applicatoins en sciences humaines. Louvain-la-Neuve: Bruylant-Academia. 
Dijst, Martin und Walter Schenkel. 2001. Urban Performance in Perspective. In: Dijst, Martin, Walter Schenkel und Isabelle Thomas (eds.). Governing Cities on the Move. Functional and Management Perspectives on Transformations of European Agglomerations. Ashgate: Aldershot. 1-18

Downs, Anthony. 1957. An Economic Theory of Democracy. New York: Harper and Brothers.

Glass, G., B. Mac Graw and M. Smith. 1994: Meta-analysis in Social Research. Beverly Hills, CA: Sage.

Goodsell, Charles T. 1994. The Case for Bureaucracy. A Public Administration Polemic. Chatham, New Jersey: Chatham House Publishers.

Guy, Simon, Robert Evans and Simon Marvin. 2000. Views of the City: Multiple Pathways to Sustainable Transport Futures. in: COST 332 - Transport and Land-Use Policies: Innovations in Institutional Arrangements for Co-ordination. Final Report of the Action. Brüssel: European Commission. S. 235-247

Haas, Ernst B. 1964. Beyond the Nation-State: Functionalism and International Organization. Stanford: Stanford University Press.

Haas, Peter M. 1992. Introduction: Epistemic Communities and International Policy Coordination. In: International Organization 1 (46). 1-35.

Hay, Colin. 2004. Theory, Stylized Heuristic or Self-Fulfilling Prophecy? The Status of Rational Choice Theory in Public Administration. In: Public Administration 1 (82). 39-62.

Heinz, Werner (Hrsg.). 2000. Stadt und Region: Kooperation oder Koordination? Ein internationaler Vergleich. Stuttgart, Berlin, Köln: Kohlhammer.

Hill, Larry B. 1991. Who Governs the American Administrative State? A Bureaucratic-Centered Image of Governance. In: Journal of Public Administration Research and Theory 3 (1). 261-294.

Holz-Rau, Christian und Ute Jansen. 2007. Nachhaltige Raum- und Verkehrsplanung. In: Aus Politik und Zeitgeschichte (29-30). 21-26.

Hunter, J. und F. Schmidt. 1990. Methods of meta-analysis. Newbury Park, CA: Sage Publications.

Janssen-Jansen, Leonie B. 2005. Beyond Sprawl: Principles for Achieving more qualitative Spatial Development. In: DISP 160 (41): 36-41.

Jones, Bryan D. 2003. Bounded Rationality and Political Science: Lessons from Public Administration and Public Policy. In: Journal of Public Administration Research and Theory 4 (13). 395-421.

Junyent, Rosa. 2000. Actors in Co-ordination for the Construction of Transport Infrastructures in Barcelona. The Delta Plan. In: COST 332 - Transport and Land-Use Policies: Innovations in Institutional Arrangements for Co-ordination. Final Report of the Action. Brüssel: European Commission. 137-151

Kaelble, Hartmut. 2002. The Historical Rise of a European Public Sphere. In: Journal of European Integration History 2 (8). 9-22.

Kaldor, Nicholas. 1939. Welfare Propositions of Economics and Inter-Personal Comparisons of Utility. In: The Economic Journal 195 (49). 549-552.

Kaufmann, Vincent und Fritz Sager. 2006. The Coordination of Local Policies for Urban Development and Public Transportation in Four Swiss Cities. In: Journal of Urban Affairs 4 (28). 353-374.

Kettl, Donald F. 2000. The global public management revolution: a report on the transformation of governance. Washington, D.C.: Brookings Institution Press.

Keating, Michael. 1995: Size, Efficiency and Democracy: Consolidation, Fragmentation and Public Choice. In: David Judge, Gerry Stoker and Harold Wolman (eds.). Theories of Urban Politics. London, Thousand Oaks, New Dehli: Sage Publications. 117-134.

Knill, Christoph. 1999. Explaining Cross-National Variance in Administrative Reform: Autonomous versus Instrumental Bureaucracies. In: Journal of Public Policy 2 (19). 113-139. 
Krücken, Georg. 2002. Amerikanischer Neo-Institutionalismus - europäische Perspektiven. In: Sociologia Internationalis (40). 227-259.

Lane, Jan-Erik. 2000. New Public Management. London, New York: Routledge.

Le Galès, Patrick. 1998. Regulation and Governance in European Cities. In: International Journal of Urban and Regional Research 3 (22). 482-506.

Lefèvre, Christian. 1998. Metropolitan Government and Governance in Western Countries: A Critical Review. In: International Journal of Urban and Regional Research 1 (22). 9-25.

Lowery, David. 1999. Answering the Public Choice Challenge: A Neoprogressive Research Agenda. In: Governance 1 (12): 22-53.

Lyons, W. E., David Lowery and Ruth Hoogland de Hoog. 1992. The Politics of Dissatisfaction. Citizens, Services, and Urban Institutions. Armonk, New York, London: M. E. Sharpe.

Margail, Fabienne and Jean-Marc Offner. 2000. The Urban Road Planning Studies: A State Procedure Dedicated to Co-ordination and Coherence. In: COST 332 - Transport and Land-Use Policies: Innovations in Institutional Arrangements for Co-ordination. Final Report of the Action. Brüssel: European Commission. 153-173

Matthiessen, Christian W. 2000. The Oresund Region. Large Scale Cross Boundary Infrastructure as a Driving Force Behind Organisational Change: Regional Integration. In: COST 332 - Transport and Land-Use Policies: Innovations in Institutional Arrangements for Co-ordination. Final Report of the Action. Brüssel: European Commission. 93-115

Morandi, Corinna, Anna Moretti, and Paola Tessitore. 2000. The Programme Agreement of Saronno-Lombardy. In: COST 332 - Transport and Land-Use Policies: Innovations in Institutional Arrangements for Co-ordination. Final Report of the Action. Brüssel: European Commission. 175-202.

Nigro, Felix A. and Lloyd G. Nigro. 1989. Modern Public Administration. $7^{\text {th }}$ edition. New York: Harper\&Row.

Nijkamp, Peter, Marc van der Burch, and Gabriella Vindigni, Gabriella. 2002. A Comparative Institutional Evaluation of Public- Private Partnerships in Dutch Urban Land-use and Revitalisation Projects. In: Urban Studies 10 (39). 1865-1880.

Niskanen, William A. 1971. Bureaucracy and Representative Government. Chicago/New York.

Obinger, Herbert. 1998. Politische Institutionen und Sozialpolitik in der Schweiz. Der Einfluss von Nebenregierungen auf Struktur und Entwicklungsdynamik des schweizerischen Sozialstaates. Frankfurt a. M. u. a.: Lang.

Osborne, David and Ted Gaebler. 1992. Reinventing Government: How the Enterpreneurial Spirit is Transforming the Public Sector. Reading.

Ostrom, Vincent, Robert L. Bish, and Elinor Ostrom. 1988. Local Government in the United States. San Francisco: Institute for Contemporary Studies.

Ostrom, Vincent und Elinor Ostrom. 1971. Public Choice: A Different Approach to Public Administration. In: Public Administration Review 2 (31). 203-216.

Powell, Walter W. and Paul J. Di Maggio (eds.). 1991. The New Institutionalism in Organizational Analysis. Chicago, London: University of Chicago Press.

Ragin, Charles C. 1987. The Comparative Method. Moving Beyond Qualitative and Quantitative Strategies. Berkely: University of California Press.

Ragin, Charles C. 2000. Fuzzy-Set Social Science. Chicago, London: University of Chicago Press.

Raphael, Lutz. 2000. Recht und Ordnung. Herrschaft durch Verwaltung im 19. Jahrhundert. Frankfurt a. M.: Fischer.

Sager, Fritz. 2002. Vom Verwalten des urbanen Raums. Institutionelle Bedingungen von Politikkoordination am Beispiel der Raum- und Verkehrsplanung in städtischen Gebieten. Bern: Haupt. 
Sager, Fritz. 2005. Metropolitan Institutions and Policy Coordination. The Integration of Land Use and Transport Policies in Swiss Urban Areas. In: Governance. An International Journal of Policy, Administration, and Institutions 2 (18). 227-256.

Scharpf, Fritz W. 1972. Komplexität als Schranke der politischen Planung. In: Politische Vierteljahresschrift, Sonderheft 4 , Gesellschaftlicher Wandel und politische Innovation ‘. 168-192.

Scharpf, Fritz W., 1991: Die Handlungsfähigkeit des Staates am Ende des zwanzigsten Jahrhunderts, in: Politische Vierteljahresschrift 32(4): 621-634.

Scharpf, Fritz W. 1992. Einführung. Zur Theorie von Verhandlungssystemen. In: Benz, Arthur, Fritz W. Scharpf und Reinhard Zintl. Horizontale Politikverflechtung. Zur Theorie von Verhandlungssystemen. Frankfurt a. M., New York. 11-28

Scharpf, Fritz W. 1993. Positive und negative Koordination in Verhandlungssystemen. in: Adrienne Héritier (Hrsg.). Policy-Analyse. Kritik und Neuorientierung. PVS Sonderheft 24. 57-83

Scharpf, Fritz W. 1994. Games Real Actors Could Play. Positive and Negative Coordination in Embedded Negotiations. In: Journal of Theoretical Politics 1 (6). 27-53.

Scharpf, Fritz W. 1997. Games Real Actors Play. Actor-Centered Institutionalism in Policy Research. Boulder, Oxford: Westview Press.

Schedler, Kuno. 1995. Ansätze einer wirkungsorientierten Verwaltungsführung. Von der Idee des New Public Management (NPM) zum konkreten Gestaltungsmodell. Fallbeispiel Schweiz. Bern, Stuttgart, Wien: Haupt.

Schimank Uwe, 2002. Organisationen. Akteurkonstellationen - korporative Akteure - Sozialsysteme. In: Jutta Allmendinger und Thomas Hinz (Hg.). Organisationssoziologie, Sonderheft 42/2002 der Kölner Zeitschrift für Soziologie und Sozialpsychologie. Opladen/Wiesbaden: Westdeutscher Verlag. 29-54.

Schneider, Carsten Q. und Claudius Wagemann. 2007. Qualitative Comparative Analysis (QCA) und Fuzzy-Sets. Ein Lehrbuch für Anwender und alle, die es werden wollen. LeverkusenOpladen: Verlag Barbara Budrich.

Scott, W. Richard and John W. Meyer. 1991. The Organization of Societal Sectors: Propositions and Early Evidence. In: Powell, Walter W. and Paul J. Di Maggio (eds.). The New Institutionalism in Organizational Analysis. Chicago, London: University of Chicago Press. 108-142.

Sessa, Carlos and Mario Gualdi. 2000: Transport and Land-Use Co-ordination: Innovative Policies in Rome. In: COST 332 - Transport and Land-Use Policies: Innovations in Institutional Arrangements for Co-ordination. Final Report of the Action. Brüssel: European Commission. 269-287.

Simon, Herbert A. 1962. The Architecture of Complexity. In: Proceedings of the American Philosophical Society 106: 467-482.

Steenberghen, Thérèse. 2000. Mobility Covenants Between Local Authorities, Public Transport Comanies and the Regional Authority in Flanders (Belgium). In: COST 332 - Transport and Land-Use Policies: Innovations in Institutional Arrangements for Co-ordination. Final Report of the Action. Brüssel: European Commission. 221-233

Tiebout, Charles M. 1956. A Pure Theory of Local Expenditures. In: Journal of Political Economy 5 (64): 416-424.

Voogd, Henk. 2000. Co-ordination Between Transport Planning and Land-Use Planning: Some Dutch Experiences. In: COST 332 - Transport and Land-Use Policies: Innovations in Institutional Arrangements for Co-ordination. Final Report of the Action. Brüssel: European Commission. 203-219

Wagschal, Uwe. 1999. Statistik für Politikwissenschaftler. München, Wien: Oldenbourg.

Weber, Max. 1972 (1922). Wirtschaft und Gesellschaft. Grundriss der verstehenden Soziologie. Tübingen: Mohr.

West, William F. 1995. Controlling the Bureaucracy. Institutional Constraints in Theory and Practice. Armonk, London: M. E. Sharpe. 
Wilson, Woodrow. 1887. The Study of Administration. In: Political Science Quarterly 2: 197-222.

Wittek, Rafael und Andreas Flache. 2002. Rational Choice und Organisationstheorie. In: Jutta Allmendinger und Thomas Hinz (Hg.). Organisationssoziologie, Sonderheft 42/2002 der Kölner Zeitschrift für Soziologie und Sozialpsychologie. Opladen/Wiesbaden: Westdeutscher Verlag. 55-87

Wolf, F.M. 1986. Meta-analysis: Quantitative methods for research synthesis. Beverly Hills, CA: Sage Publications.

Wolf, Patrick J. 1993. A Case Survey of Bureaucratic Effectiveness in U.S. Cabinet Agencies: Preliminary Results. In: Journal of Public Administration Research and Theory 2 (3). 161-181.

Zucker, Lynne G. 1983. Organizations as Institutions. In: S. B. Bachrach, (ed.). Research in the Sociology of Organizations. Greenwich, Conn.: JAI Press. 1-42 